\title{
LOCALIZATIONS IN UNIVERSAL TOPOLOGICAL CATEGORIES
}

\author{
F. CAGLIARI AND S. MANTOVANI
}

(Communicated by Dennis Burke)

\begin{abstract}
For some familiar topological categories it is shown that the subcategory of indiscrete spaces is the only nontrivial localization.
\end{abstract}

Introduction. The notion of localization (=left exact reflection) plays an important role in the theory of sheaves and of topoi. It has been studied in detail even for general categories [2], but applications are mostly given for abelian or at least additive categories. The purpose of this paper is to show that in the categories $\mathscr{T}_{0} \rho$ of topological spaces, $\mathscr{R}_{0}$ of $R_{0}$-topological spaces, $\mathscr{C} \mathscr{R} e g$ of completely regular spaces and $\mathscr{P}_{\text {rac }}$ of proximity spaces, the subcategory $\mathscr{I}_{\text {nd }}$ of indiscrete spaces is the only nontrivial localization, that is, the only (full and replete) reflective subcategory whose reflector preserves finite limits. In fact, except for $\mathscr{R}_{0}$, preservation of regular monomorphisms suffices for this result.

We refer the reader to [4 and 5] for notation and definitions not explicitly given here.

1. THEOREM. The only nontrivial localization of $\mathscr{T}_{\mathrm{p}} \mathrm{p}$ is $\mathscr{I}_{\mathrm{n}}$.

Proof. Let $\mathscr{C}$ be a localization of $\mathscr{T}_{o p}, \mathscr{C} \neq \mathscr{T}_{0}, \mathscr{C} \neq \mathscr{S}_{\text {ing }}$. $\mathscr{C}$ is bireflective in $\mathscr{T}_{0} \rho$ because the $\mathscr{C}$-reflection preserves monomorphisms (cf. [6]), so the Sierpinski space $\mathbf{S}$ cannot belong to $\mathscr{C}$ since $\mathscr{C} \neq \mathscr{T}_{0}$. Hence the $\mathscr{C}$-reflection of the Sierpinski space is $\mathbf{I}_{2}$ (the two-point indiscrete space), and the $\mathscr{C}$-reflection of $\mathbf{S} \times \mathbf{S}$ is the four-point indiscrete space as the $\mathscr{C}$-reflection preserves products. The space $\mathbf{D}_{2}$ (the two-point discrete space) can be embedded into the complement of the diagonal of $\mathbf{S} \times \mathbf{S}$ and so, since the $\mathscr{C}$-reflection preserves regular monomorphisms, the $\mathscr{C}$-reflection of $\mathbf{D}_{2}$ must be $\mathbf{I}_{2}$. Therefore, $\mathscr{C}$ must be $\mathscr{I}_{\text {nd }}$.

This result can be extended to some universal topological categories as we are going to prove next.

2. THEOREM. Let $\mathscr{A}$ be a universal topological category in which connectedness are productive, and $\mathscr{C}$ be a localization of $\mathscr{A}$. We have:

(a) The connectedness related to $T_{0} \mathscr{C}$ (cf. [4]) is epireflective in $\mathscr{A}$.

(b) The quotient reflective hull $\mathbf{Q}\left(T_{0} \mathscr{C}\right)$ of $T_{0} \mathscr{C}$ is a disconnectedness.

Proof. (a) $X$ is $T_{0} \mathscr{C}$-connected if and only if the $T_{0} \mathscr{C}$-reflection of $X$ is a singleton (cf. [4]), and since $\mathscr{A}$ is universal, $X$ is $T_{0} \mathscr{C}$-connected if and only if the $\mathscr{C}$-reflection of $X$ is indiscrete. So as the $\mathscr{C}$-reflection preserves regular monomorphisms, we have that the category of $T_{0} \mathscr{C}$-connected spaces is productive and hereditary; that is, it is epireflective in $\mathscr{A}$ (cf. [3]).

Received by the editors September 15, 1986 and, in revised form, February 23, 1987.

1980 Mathematics Subject Classification (1985 Revision). Primary 54B30, 18A40.

This work was supported by funds of M.P.I. (40\%), Italy. 
(b) Suppose $X$ is $T_{0} \mathscr{C}$-totally disconnected and $X$ does not belong to $\mathbf{Q}\left(T_{0} \mathscr{C}\right)$. Then the $T_{0}$-reflection $r: X \rightarrow r X$ is not injective; that is, there is a point $x$ of $r X$ such that $r^{-1}(x)$ is not a singleton. Since $\mathscr{A}$ is universal, the $\mathscr{C}$-reflection $r^{\prime}: X \rightarrow r^{\prime} X$ is such that the structure on $r^{\prime}\left(r^{-1}(x)\right)$ induced by $r^{\prime} X$ is indiscrete. As the $\mathscr{C}$-reflection preserves regular monomorphisms, the $\mathscr{C}$-reflection of $r^{-1}(x)$ is an indiscrete space. Hence $r^{-1}(x)$ is a $T_{0} \mathscr{C}$-connected space with more than one point; but this is absurd, as $X$ is hereditary $T_{0} \mathscr{C}$-disconnected.

3. COROLlaRy. The only nontrivial localization of $\mathscr{P}_{\text {rox }}, \mathscr{R}_{1}$ and $\mathscr{C} \mathscr{R}$ eg is Ind.

PROOF. In any of these categories there are only two structures on a set with two points: the discrete one and the indiscrete one. Therefore, if a localization $\mathscr{C}$ of any of these categories is different from $\mathscr{I}_{n d}$, the two-point discrete space $\mathbf{D}_{2}$ is in $\mathscr{C}$, even in $T_{0} \mathscr{C}$. Therefore, by Theorem 2 , the category of $T_{0} \mathscr{C}$-connected spaces is an epireflective subcategory which does not contain $\mathbf{D}_{2}$. So it must be Ind . Thus the disconnectedness related to $T_{0} \mathscr{C}$, that is $\mathbf{Q}\left(T_{0} \mathscr{C}\right)$ by Theorem 2 , must be $T_{0} \mathscr{P}_{r a x}, T_{0} \mathscr{R}_{0}=\mathscr{T}_{1}$ and $T_{0} \mathscr{C} \mathscr{R}$ eg $=\mathscr{T}_{y c h}$, respectively (cf. [4, Proposition 1]). But in any of these categories this means that $\mathscr{C}$ must be the whole category.

4. REMARK. We can observe that Theorem 2 still works for reflective (not only bireflective) subcategories whose reflection preserves regular monomorphisms. (Recall that when $\mathscr{C}$ is reflective and not bireflective, $T_{0} \mathscr{C}=\mathscr{C}$.) Using arguments quite similar to the ones exposed earlier, we can state:

4.1. The only nontrivial reflective subcategory $\mathscr{B}$ of $\mathscr{T}_{0}, \mathscr{P}_{\text {rox }}$, and $\mathscr{C} \mathscr{R}$ eg preserving regular monomorphisms is Ind.

In fact, if $\mathscr{K}$ is any of these categories, the connectedness $\mathbf{C}(\mathscr{B})$ related to $\mathscr{B}$ must be epireflective, by Theorem 2 . So there are only three possibilities: (i) $\mathbf{C}(\mathscr{B})=\mathscr{S}$ ing, (ii) $\mathbf{C}(\mathscr{B})=$ Ind and (iii) $\mathbf{C}(\mathscr{B})=\mathscr{K}$. In case (i), we have $\mathbf{Q}(\mathscr{B})=\mathscr{K}$, but this is not possible, as $\mathscr{B} \subset T_{0} \mathscr{K}$ and then $\mathbf{Q}(\mathscr{B})$ must be contained in $T_{0} \mathscr{K}$. In case (ii) it follows that $\mathbf{Q}(\mathscr{B})=T_{0} \mathscr{K}$ and then $\mathscr{B}=$ $T_{0} \mathscr{K}$. But this is not possible, as regular monomorphisms of $T_{0} \mathscr{T}_{0} \rho, T_{0} \mathscr{P}_{\text {rox }}$, and $T_{0} \mathscr{C} \mathscr{R}$ g g do not coincide with the embeddings. So it only remains case (iii), which implies $\mathscr{B}=$ Ind .

4.2. The only nontrivial reflective subcategories of $\mathscr{R}_{0}$ preserving regular monomorphisms are $\mathscr{I}_{\text {nd }}$ and $\mathscr{T}_{1}=T_{0} \mathscr{R}_{0}$. In fact, the reflection of $\mathscr{R}_{0}$ in $\mathscr{T}_{1}$ preserves regular monomorphisms, so also case (ii) is possible.

\section{REFERENCES}

1. F. Cagliari and S. Mantovani, On disconnectedness in subcategories of a topological category and related topics, Suppl. Rend. Circ. Mat. Palermo 12 (1986), 205-212.

2. C. Cassidy, M. Hebert, and G. M. Kelley, Reflective subcategories, localizations and factorization systems, J. Austral. Math. Soc. Ser. A 38 (1985), 287--329.

3. H. Herrlich and G. Strecker, Category theory, Heldermann-Verlag, Berlin, 1979.

4. T. Marny, On epireflective subcategories of topological categories, General Topology Appl. 11, (1980), 175-181.

5. G. Preuss, Relative connectedness and disconnectednesses in topological categories, Quaestiones Math. 2 (1977), 297306.

6. C. M. Ringel, Monofunctors as reflectors, Trans. Amer. Math. Soc. 161 (1971), 293-306.

Dipartimento di Matematica, Università di Parma, Via Università 12,43100 PARMA, ITALY 